\title{
Programmable tandem detonator
}

\section{Tomasz Kuczerski, Zbigniew Lewandowski, Dariusz Gibalski}

Tomasz Kuczerski, Zbigniew Lewandowski, Dariusz Gibalski, "Programmable tandem detonator," Proc. SPIE 11442, Radioelectronic Systems Conference 2019, 1144216 (11 February 2020); doi: 10.1117/12.2565066

SPIE. Event: Radioelectronic Systems Conference 2019, 2019, Jachranka, Poland 


\title{
Programmable tandem detonator
}

\author{
Tomasz Kuczerski, Zbigniew Lewandowski, Dariusz Gibalski \\ Military Institute of Armament Technology 05-220 Zielonka, Poland
}

\begin{abstract}
The paper presents a practically realized system of electronic programmable detonator designed for tandem systems. The presented layout was implemented on the basis of micro-controllers using RISC architecture. The detonator is equipped with a fast precursor excitation system and can be programmed for the required delay time of the detonation of the main charge in the range from a few to several hundred $\mu$ s. The paper presents the results of laboratory preliminary tests of the implemented system. Thanks to the possibility of delay time programming, the system has a wide range of practical applications also during shooting tests.
\end{abstract}

Keywords: programmable detonator, tandem system, micro-controller.

\section{INTRODUCTION}

The design of new cumulative missiles with precursors in tandem system [1] capable of destroying reactive armour [2] requires the development of appropriate ignition systems [3]. The principle of tandem projectiles is based on a separate stimulation of the precursor level and the main charge. The precursor is usually stimulated with a minimum delay time. The main charge must be excited within a precisely defined time interval due to the design of the projectile and its velocity.

The construction of tandem detonators and the construction of safe mechanisms to arm them [4] is a wide area of expertise, which is difficult to put into this article in its entirety. Basically, a tandem detonator is simply a double detonator where the distance between the first and second charge provides a puncture in the reactive armour. International regulations for this type of construction regulate and limit the chemicals used to build detonators (primers). Therefore, weaker substances [5] from secondary material according to STANAG standards are used. They require special stimulation with an electric fuse $[5,6,7]$. This is why this two-stage processor-controlled fuse with an electric actuator was designed [8].

When testing the effectiveness of armour piercing in polygonal conditions, it is often necessary to change the delay time of detonation of the main charge in relation to the precursor. The use of a programmable system significantly simplifies and speeds up the execution of a series of tests and allows to reduce the costs of tests. The aim of this study is to present the designed and constructed tandem system of a programmable electronic detonator and to present the results of laboratory preliminary tests.

\section{PRINCIPLE OF OPERATION OF THE SYSTEM}

Figure 1 shows the block diagram of the programmable electronic tandem detonator. The detonator uses three microcontrollers (processors in RISC architecture), a stable clock generator, a voltage stabilizer, a comparator and thyristor TH-1 and TH-2 executive circuits to stimulate primers.

The main processor (Fig. 1, micro-controller 3) performs the control and communication functions of the master system. For information transmission it uses serial RS communication and internal parallel data bus.

The two processors of the precursor and main charge circuits (Figure 1, micro-controller 1,2) are directly connected by an internal data bus to a common clock signal. This enables the software to synchronize the operation of all three processors that operate in quasi-real-time mode. Synchronization ensures that the minimum delay times resulting from the algorithm implementation on a three-processor system are achieved.

Two signals are supplied to the comparator input: a reference signal (constant voltage) and a signal from an analog force sensing resistor (FSR), which is an impact sensor in this application. At the moment of impact the resistance of the

Radioelectronic Systems Conference 2019, edited by Piotr Kaniewski, Jan Matuszewski, Proc. of SPIE

Vol. 11442, $1144216 \cdot$ (c) 2020 SPIE · CCC code: 0277-786X/20/\$21 · doi: 10.1117/12.2565066 
sensor changes, which results in a change of voltage on the comparator INP input. The actuating speed of the used comparator is about a few $\mu$ s (Fig. 4). This signal can directly control the precursor primer excitation system by means of protection systems without additional delays. There is a possibility of introducing additional delay in micro-controller 1 circuit (Fig. 1) but it is not used in practice. Tandem missiles usually detonate the precursor charge immediately.

The signal from the mass comparator output is simultaneously transmitted to the second micro-controller, which counts down the programmed delay time and controls the TH-2 main charge primer excitation system. The high effectiveness of the reactive armour piercing [1] is achieved by the main charge activation delay, appropriate to the cumulative missile velocity.

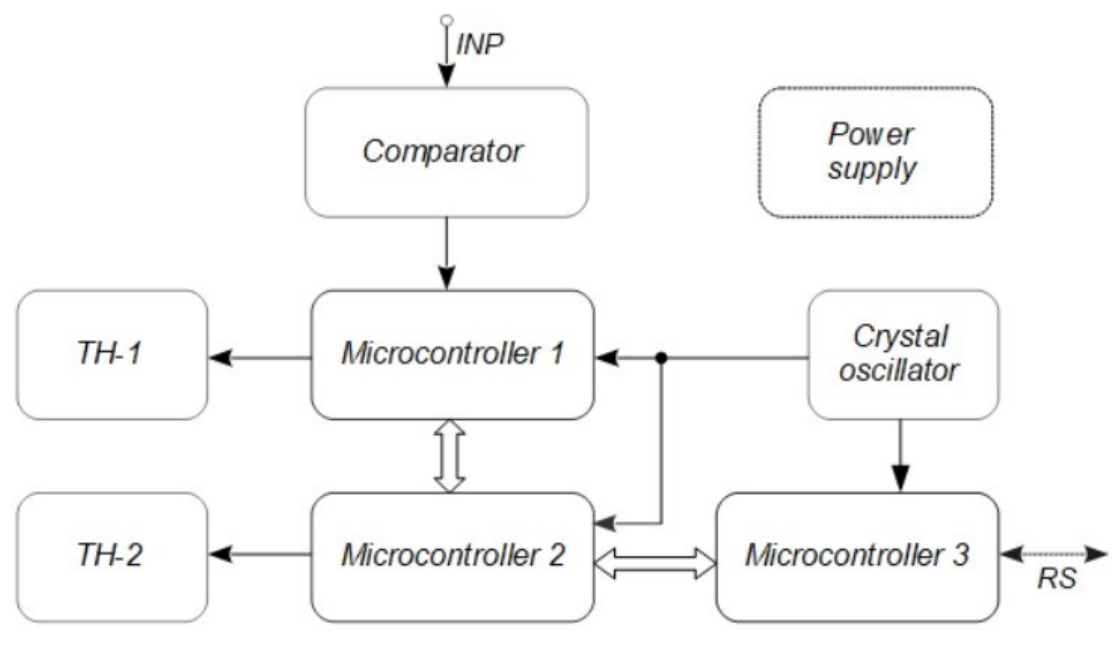

Figure 1. Block diagram of the programmable electronic tandem detonator.

The generalised algorithm of the system is shown in Fig. 2. It is implemented mostly on the main processor (Fig. 1, micro-controller 3). Two auxiliary processors were used to independently start the TH-1 and TH-2 executive systems. The first one was used to operate the comparator and the second one to delay the excitation channel of the main charge TH-2.

The initial state of the POWER/RESET system occurs at the moment of power supply OFF/ON or enforced RESET state. The next START state includes the initial procedures (setup), responsible for the initial settings and test procedures, responsible for the safety of the system. In the event of a system failure (e. g. a failure of protective systems), it shall be locked and returned to the ERROR state until the POWER/RESET state occurs.

If the system is operational, it reaches the READY state. In this state, the system can receive control (programming) commands along the RS line that change the stored settings in the EEPROM memory, e. g. to modify the required delay time for excitation of the main charge.

The initial settings and test procedures depend on the system parameters stored in the EEPROM. When programming the detonator, specific data blocks are stored in EEPROM memory, including a parameter that determines the delay time of the detonation of the main charge. Several independent processor memory areas are used to store the same data blocks to increase reliability.

In the READY state, after fulfilling the arming conditions (e. g. after a specified time or for a command transmitted via the RS line), the system reaches the ARMED arming state. If any of the conditions for safe arming are not met, the system is blocked until the POWER/RESET state occurs.

In the ARMED state, after triggering the excitation system or (in laboratory conditions) after giving the INP pulse to the comparator input (fig. 1, 4), the TH-1 precursor charge primer detonates.

The delay time of precursor detonation is minimal $(<5 \mu \mathrm{s})$, it can be programmed as longer by introducing additional delay inside micro-controller 1 (Fig. 1), but in most applications only main charge detonation delay is used [2]. 


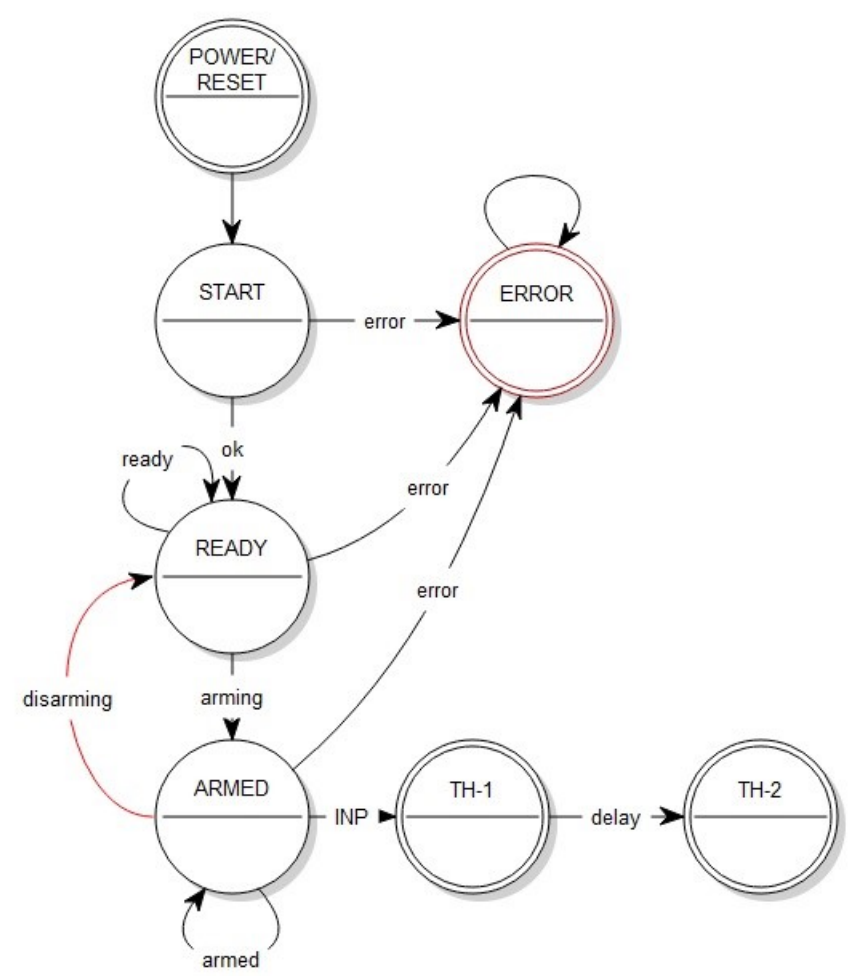

Figure 2. State machine algorithm of a programmable tandem detonator.

The TH-2 main charge primer detonates after the programmed delay time has elapsed. The micro-controller 2 is responsible for this delay.

The system has the ability to stimulate low and high voltage primers. The type of used primers can be programmed in EEPROM memory.

Depending on the type of applied protections (including mechanical protections) [3], selected outputs intended for triggering primers can also be programmed to be activated at the moment of ARMED state occurrence (e. g. to signal the status of the system or to unblock mechanical protections).

The above functionality of the programmable system allows to carry out various types of one-time or repeatable experiments that occur during the prototyping phase of missiles.

\section{LABORATORY RESEARCH}

A preliminary laboratory test stand consisting of a functional generator, an oscilloscope, a power supply unit and a tested system is shown in Fig. 3.

The main purpose of the preliminary tests was to check the functioning of the system, verify the design assumptions and measure the delay time at the exit to detonation of the main charge primer cap in relation to the impulse at the input of the comparator $[9,10]$. The system, during this tests, was programmed for a delay of $110 \mu \mathrm{s}$.

The excitation system was replaced by an impulse from a function generator, which was fed to the INP input of the comparator of the programmable tandem detonator. The impulse parameters have been selected so that it causes the comparator to trip (Fig. 1), similarly as in the case of the impact sensor trip. 
The system uses a strain gauge as an impact sensor. Its characteristics allow to obtain very high speed triggering of the comparator system. The total delay of the sensor and comparator is less than a few $\mu \mathrm{s}$.

In systems of tandem detonators with a precursor, it is very important to achieve a minimum firing time of the charge [2]. The precursor must act very quickly. His job is to eliminate the reactive armor protection. The time delay of the main charge is measured only after it has been triggered. An important structural element of the system is to maintain the functionality of the delay system after the detonation of the precursor. It consists in the fact that the precursor charge cannot damage the electronic system responsible for measuring the delay time.
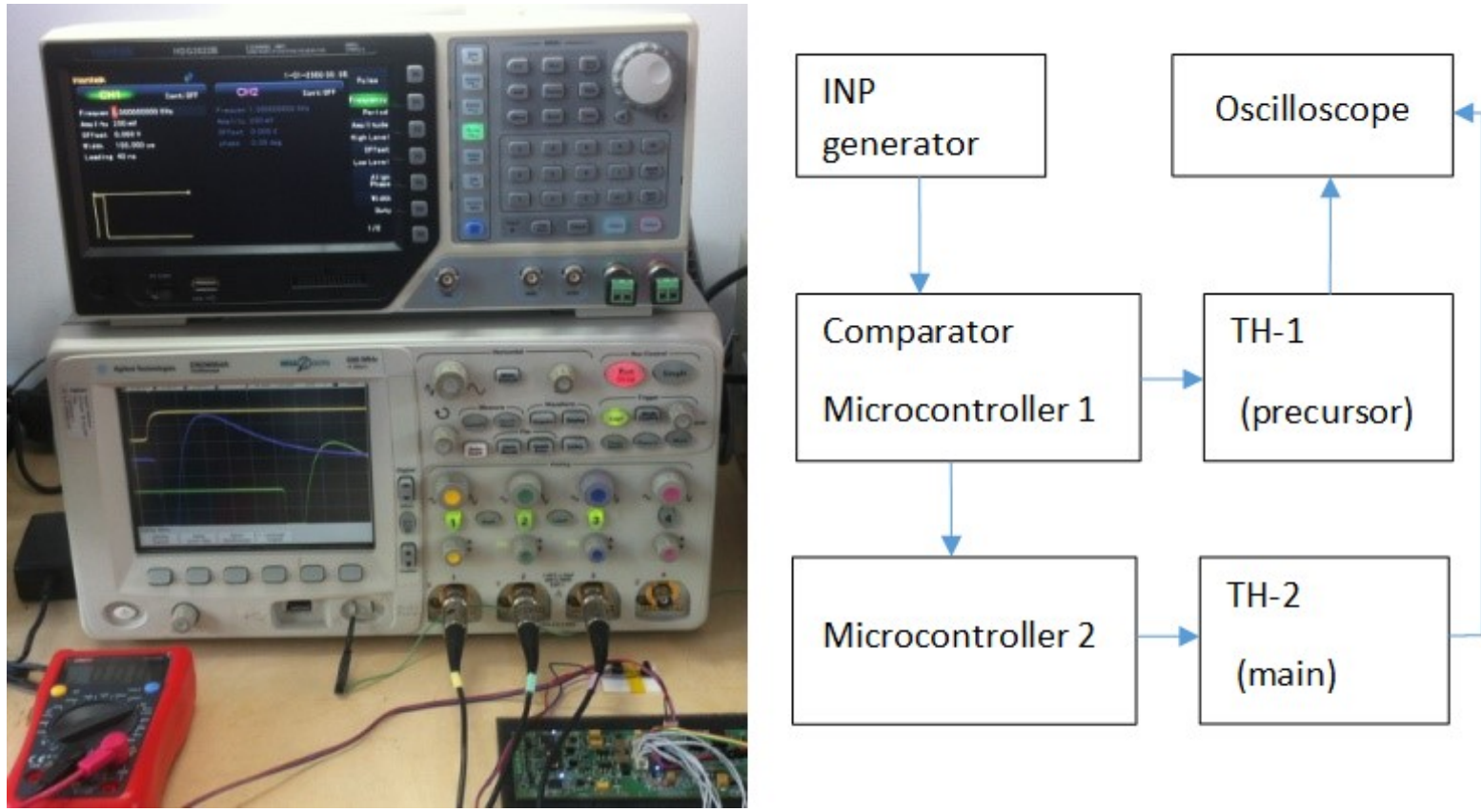

Figure 3. Laboratory test rig for programmable tandem detonator.

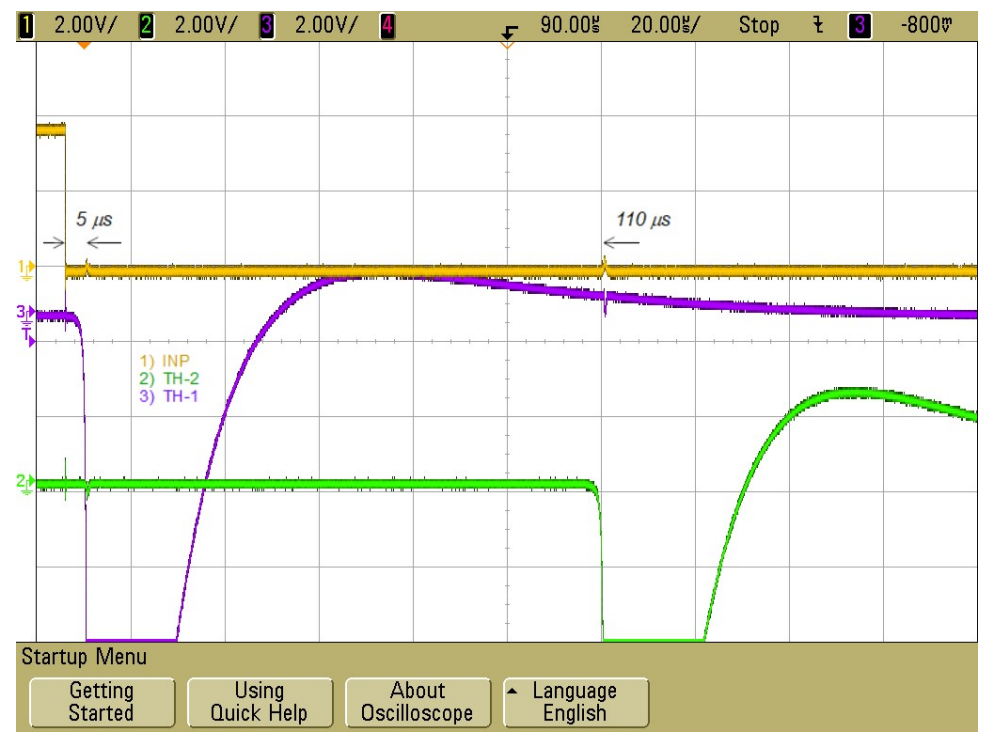

Figure 4. Measurement of delay time(yellow - imp. from a generator, equivalent to an impulse from an impact sensor, violet - activation of the TH-1 precursor detonation system, green - delayed activation of the TH-2 main charge system). 
On the oscilloscope (Fig. 4) was observed: 1) an INP generator pulse, 2) $110 \mu$ s delayed main charge (TH-2) primer excitation pulse, 3) a precursor primer excitation pulse.

In order to test the practical programmable tandem detonator (Fig. 3), a resistance pressure sensor (impact sensor strain gauge) working in the range of up to several hundred $\mathrm{N}$ was connected (as an analogue excitation system).

A series of tests was performed that involved hitting a pressure sensor to simulate its action. During the simulation of the impact, the operation of the system was observed. Changing the resistance of the strain gauge caused a change of voltage at the input of the comparator. As a result, the comparator was activated. Examples of two attempts are shown in Fig. 5.

On the oscilloscope (Fig. 5) was observed: 1) change of voltage as a result of a change of resistance of the sensor, 2) impulse of excitation of the primer of the main charge, 3) impulse of excitation of the primer of the precursor. An important safety element of the system is that in the READY state (Fig. 2) the comparator cannot be switched on. It means the accidental impact won't detonate. To perform this check, the system was set to READY and subjected to impact tests. Only after the system was put into the ARMED state, the comparator could be activated. During the tests, the repeatability of the delay circuit was also checked. It is dependent on the clock frequency and this depends on the temperature. In order to eliminate this phenomenon, a temperature stabilized clock signal source has been used.
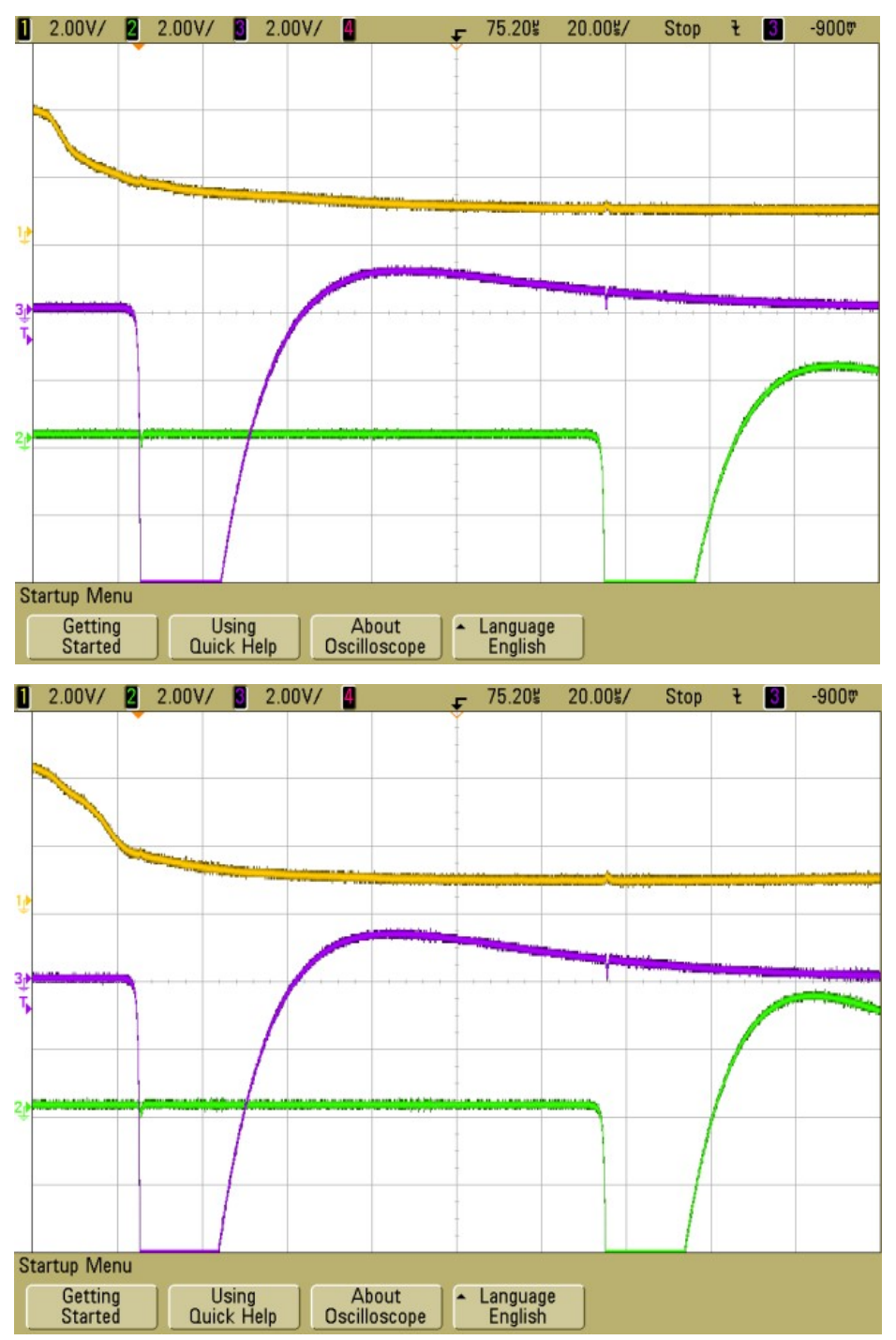

Figure 5. Measurement of delay time with resistance pressure sensor, 2 tests (yellow - imp. from a generator, equivalent to an impulse from an impact sensor, violet - activation of the TH-1 precursor detonation system, green - delayed activation of the TH-2 main charge system). 


\section{CONCLUSIONS}

Laboratory tests confirmed the design assumptions of the system and showed a delay of excitation of the main charge in accordance with the programmed delay. Multiple attempts have shown the repeatability of the programmed delay time. No faulty operation was observed in a wide temperature range. The system was subjected to destructive tests during which the delay time was checked. There are several versions of the structure in terms of the arrangement of discrete elements. During the research, some structural shortcomings were also noted, including errors in the placement of elements on the board, which caused the appearance of small disturbing impulses when the precursor primer cap is excited (they are visible on the oscilloscope in the main charge excitation channel in Fig. 4,5). The amplitude of these disturbances was too small to cause an accidental, parallel excitation of the primer in the second channel. The results of the tests allowed for the removal of the defects. In the next version of the PCB the channel separation has been improved and even separate power supply voltage stabilization systems have been applied.

Laboratory and field tests will be continued for the next PCB versions. Due to the high functionality of the tandem detonator, which has two separate channels and an additional processor for communication and control the system can also be used as a single-channel programmable detonator.

The main charge primer excitation system has been practically tested on a training ground using a target primer and a small charge.

Research has shown that the programmable detonator is very useful for testing new missile structures during the field shooting research at our institute. New experience has led to the development of better and safer designs for tandem detonators.

\section{REFERENCES}

[1] Wiśniewski A., Podgórzak P.: Research results on precursor of tandem shaped charge projectile model. Military Institute of Armament Technology (2005).

[2] Wiśniewski A.: Technological problems encountered during the elaboration of a warhead component with a diaphragm. Military Institute of Armament Technology, Central European Journal of Energetic Materials, (2013).

[3] Derecki S., Stor E.: Basics of artillery detonators design, Military University of Technology (1983).

[4] Popescu Gh; Ghimiş Ştefan; Stancioiu Alin. The results of the researches reguarding the use of cams mechanisms in assembling electrical detonators. Annals of the Oradea University- Fascicle of Management and Technological Engineering, Volume VII (XVII), 2008, Editura Universităţii din Oradea, pag. 1040.

[5] Fousson, Eric \& Ritter, Auguste \& Arnold, Thomas. (2016). High Safety and Reliability Electric Detonator. Propellants, Explosives, Pyrotechnics. 41. 10.1002/prep.201500310.

[6] Varesh, Ron. (1996). Electric Detonators: EBW and EFI. Propellants, Explosives, Pyro-technics. 21. 150 - 154. 10.1002/prep.19960210308.

[7] Solov'ev, V. \& Ovchinnikov, N. \& Patsyuk, V. \& Lavrov, V.. (2015). A new generation of special electric detonators. Journal of Machinery Manufacture and Reliability. 44. 726-736. 10.3103/S1052618815080051.

[8] Varosh, R. (1996). Electric detonators: EBW and EFI. Propellants Explosives Pyrotechnics. 21. 150-154.

[9] Yankovskiy, Boris. (2011). Conditions of Experimental Realization of an Electric Detonation. 1020.

[10] Hurley, Eldon \& Kome, Cornelis \& Levan, Robert. (2013). Method and system for communicating and and controlling electric detonators. US8390979B2. 